\title{
Microbunch dynamics and multistream instability in a heavy-ion synchrotron
}

\author{
Sabrina Appel ${ }^{1}$ and Oliver Boine-Frankenheim ${ }^{1,2}$ \\ ${ }^{1}$ GSI Helmholtzzentrum für Schwerionenforschung, Planckstraße 1, 64291 Darmstadt, Germany \\ ${ }^{2}$ Technische Universität Darmstadt, Schlossgartenstraße 8, 64298 Darmstadt, Germany
}

(Received 14 February 2012; published 17 May 2012)

\begin{abstract}
For the operation as an injector for the FAIR project, the heavy-ion synchrotron (SIS) has to deliver intense and high quality ion bunches with a high repetition rate. One requirement is that the initial momentum spread of the injected coasting beam should not exceed the limit set by the available SIS rf bucket area. Furthermore, the Schottky spectrum should be used to routinely measure the momentum spread and revolution frequency directly after injection in order to adjust the rf settings. During the transverse multiturn injection, the SIS is filled with microbunches from the UNILAC linac at $36 \mathrm{MHz}$. At low beam intensities, the microbunches debunch within a few turns and form a coasting beam with a Gaussian-like momentum distribution. With increasing intensity we observe persistent current fluctuations and an accompanying broadband, pseudo-Schottky spectrum. Analytical and numerical models indicate that the space charge induced multistream instability of the filaments formed after debunching is responsible for the observed turbulent current spectrum.
\end{abstract}

DOI: 10.1103/PhysRevSTAB.15.054201

PACS numbers: 29.20.D-, 29.27.Ac, 52.65.Rr

\section{INTRODUCTION}

Collective effects induced by longitudinal space charge in intense coasting or bunched ion beams are important in many storage rings or synchrotrons. Longitudinal space charge below the transition energy affects the stability thresholds and can lead to persistent current modulations in coasting beams [1]. Longitudinal space charge can be responsible for a sustained linac microbunch structure after injection into a synchrotron or storage ring with no rf. This has been observed in the Los Alamos proton storage ring (PSR) [2]. In [3] it was pointed out that during the debunching of linac microbunches a fast multistream instability can occur, which develops a turbulent frequency spectrum ("pseudo-Schottky" signal). The interpretation of the beam fluctuation spectrum right after injection is important for the fast measurement of the momentum spread. The space charge induced fluctuations might also possibly lead to an undesired beam quality reduction.

In the heavy-ion synchrotron (SIS) at GSI, intense heavy-ion microbunches are injected with $36 \mathrm{MHz}$ from the UNILAC at $11.4 \mathrm{MeV} / \mathrm{u}$ for up to 20 turns [4]. For the SIS intensity upgrade program, the resulting rms momentum spread of the debunched beam has to remain below a maximum value determined by the available rf bucket area for fast ramping [5]. Furthermore, the momentum spread before rf capture should be measured within a few ms using the Schottky spectrum from the

Published by the American Physical Society under the terms of the Creative Commons Attribution 3.0 License. Further distribution of this work must maintain attribution to the author(s) and the published article's title, journal citation, and DOI. coasting beam. In the present study we compare analytical and simulation models of the multistream instability with the measured signals from the injected microbunches in the SIS.

\section{LONGITUDINAL SPACE CHARGE}

During multiturn injection from a linac into a synchrotron, the linac microbunches are injected with a transverse offset (see e.g. [6], page 214). Afterwards the injected microbunches expand and overlap in the longitudinal plane. In this section we will justify our one-dimensional model for the longitudinal space charge field of the microbunches injected into a ring. In the SIS the initial half length of the injected microbunches is in the range of $z_{m} \approx 0.1-0.3 \mathrm{~m}$. The bunch length is still larger than the average radius of the SIS beam pipe $(b \approx 0.1 \mathrm{~m})$. For bunches much longer than the cutoff wavelength of an ideally conducting pipe, we can calculate the longitudinal electric field on the beam axis from

$$
E_{z}=-\frac{q g}{4 \pi \epsilon_{0} \gamma_{0}^{2}} \frac{\partial \lambda}{\partial z}
$$

where $q$ is the charge, $\epsilon_{0}$ the vacuum permittivity, $\gamma_{0}$ the relativistic parameter, $z$ the longitudinal coordinate, and $\lambda(z)$ the line density of the beam. The $g$ factor is given through

$$
g=1+2 \ln \frac{b}{a}
$$

where $b$ is the pipe radius and $a$ the beam radius. This expression for the $g$ factor can be extended to beams with a transverse offset $d$ (see the Appendix). In this case we obtain 


$$
g(d)=1+2 \ln \left[\frac{b+d x_{d}}{a}\right]
$$

with

$$
x_{d}=-\frac{d}{b} .
$$

The offset $d$ enters only in the logarithm. If a microbunch is injected with an offset, we will assume in the following that the longitudinal electric field on the beam axis can still be obtained from the centered $(d=0)$ bunch. The exact longitudinal electric field for a microbunch in an ideally conducting beam pipe can only be obtained numerically from the solution of the Poisson equation in the beam frame (see also Ref. [7], page 402). We use the three-dimensional Poisson solver from the commercial simulation tool CST EM STUDIO ${ }^{\circledR}$ in order to obtain the longitudinal electric field for a microbunch with a parabolic line density. The resulting electric field on the beam axis is shown in Fig. 1 together with the field obtained from Eq. (1). For a parabolic bunch profile, the space charge field is linear inside the bunch and vanishes outside. It can be seen that only at the bunch edges the numerically obtained field deviates from the approximation. For different bunch lengths, one can approximate the numerical electric field near the bunch center by a linear curve with a corresponding $g$ factor. The resulting plot is shown in Fig. 2. It can be observed that the approximation of a constant $g$ factor applies for $z_{m} / a \gtrsim 10$.

For shorter bunches and for small wavelengths, one has to take into account that the contribution of image currents in the beam pipe to the longitudinal electric field disappears for wavelengths below (Ref. [8])

$$
\lambda_{c} \approx \gamma_{0}^{-1} \pi a \sqrt{g} \text {. }
$$

For arbitrary wavelengths the space charge impedance can be approximated very well through

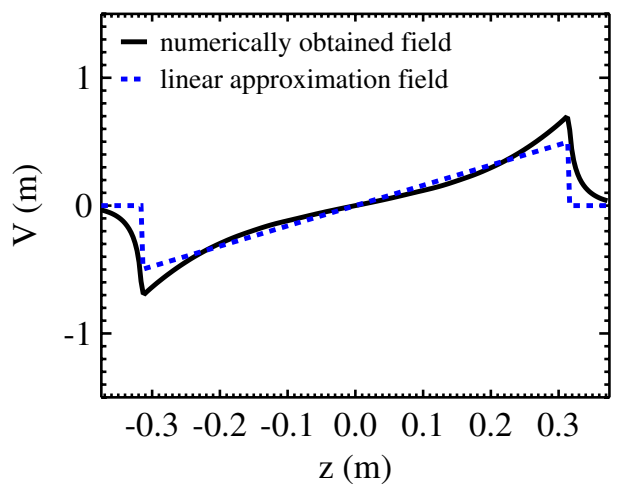

FIG. 1. The longitudinal electric field for a bunch with a parabolic line density with $z_{m} / a=9$ and $a=3.5 \mathrm{~cm}$. The plot shows the numerically obtained field (solid curve) together with the linear approximation (dashed blue curve).

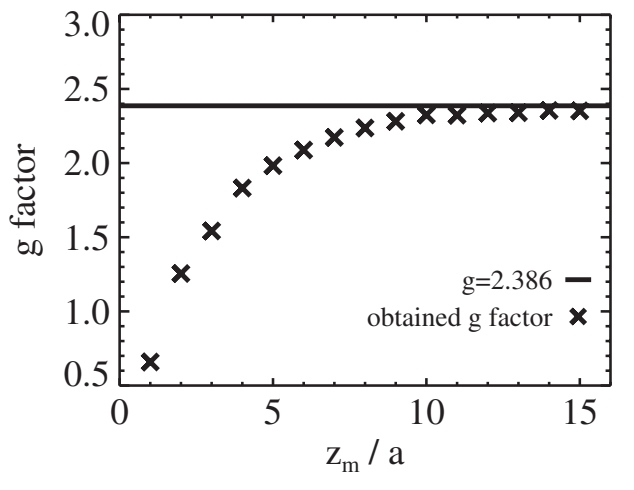

FIG. 2. The $g$ factor obtained from the numerical electric field (crosses) as a function of the bunch length. It can be seen that the approximation of a constant $g$ factor (solid line) applies for $z_{m} / a \gtrsim 10$.

$$
\frac{Z_{n}^{\mathrm{sc}}}{n}=-i \frac{Z_{0} g}{2 \beta_{0} \gamma_{0}^{2}} \frac{1}{1+\left(\frac{n}{n_{c}}\right)^{2}},
$$

where $n_{c}$ is the cutoff harmonic number

$$
n_{c}=2 \pi R / \lambda_{c},
$$

$Z_{0}$ is the vacuum impedance, $\beta_{0}$ the relativistic factor of the beam, and $R$ the ring radius ( $R=34 \mathrm{~m}$ for the SIS). The electric field can be obtained from the Fourier components of the line density $\lambda_{n}$ and the impedance $Z_{n}$. In the following we will use Eq. (1) in order to describe the longitudinal space charge field of injected microbunches before they start to overlap with their adjacent bunches.

\section{MICROBUNCH DYNAMICS: SHORT TERM}

In the SIS the injected microbunches debunch within a few turns and form a coasting beam because there is no $\mathrm{rf}$ system with the corresponding frequency $(36 \mathrm{MHz})$. As long as adjacent bunches do not overlap, we can describe the debunching process using the longitudinal envelope equation in a synchrotron [9],

$$
z_{m}^{\prime \prime}-\frac{K_{L}}{z_{m}^{2}}-\frac{\epsilon_{L}^{2}}{z_{m}^{3}}=0
$$

with the longitudinal perveance

$$
K_{L}=\frac{3 g N_{b}\left(Z^{2} / A\right) r_{p} \eta_{0}}{2 \beta_{0}^{2} \gamma_{0}^{3}}
$$

following from the linear electric field given by Eq. (1). $N_{b}$ is the number of ions in the bunch, $Z$ the charge number of an ion with mass number $A, r_{p}=e^{2} /\left(4 \pi \epsilon_{0} m_{p} c^{2}\right) \approx$ $1.53 \times 10^{-18} \mathrm{~m}$ the proton radius, $m_{p}$ the proton mass, $c$ the speed of light, $e$ the electron charge, and $\epsilon_{L}=$ $\left|\eta_{0}\right| z_{m} \delta_{0}$ the longitudinal emittance with the slip factor $\eta_{0}=1 / \gamma_{t}^{2}-1 / \gamma^{2}$ and the transition energy $\gamma_{t} . \delta_{0}=$ $\delta p / p_{0}$ is the maximum momentum deviation for an upright ellipse. Here we follow the notation of Refs. [7,10]. 
The ratio of the second term (space charge term) and the third term (emittance term) of Eq. (7) is the dimensionless space charge parameter for bunched beams,

$$
\Sigma=\frac{2 K_{L} z_{m}}{\epsilon_{L}^{2}} .
$$

Multiplying Eq. (7) by $z_{m}^{\prime}$ and integrating it with respect to $s$, we obtain a longitudinal invariant,

$$
I_{L}=\frac{z_{m}^{\prime 2}}{2}+\frac{K_{L}}{z_{m}}+\frac{\epsilon_{L}^{2}}{2 z_{m}^{2}} .
$$

The first term of Eq. (10) is equivalent to the coherent kinetic energy, the second term to the space charge energy, and the third term to the incoherent kinetic energy.

For a rotated ellipse in Fig. 3 in the plane $\left(z, \delta=\delta p / p_{0}\right)$ $\delta_{m}=\left(\delta p / p_{0}\right)_{m}$ is the momentum offset of the bunch ends and $\delta_{0}=\left(\delta p / p_{0}\right)_{0}$ the maximum momentum deviation in the bunch center. The total momentum spread $\Delta=\Delta p / p_{0}$ for a rotated ellipse by using $\Delta^{2}=\delta_{0}^{2}+\delta_{m}^{2}$ is then [10]

$$
\Delta=\sqrt{\left(\frac{\epsilon_{L}}{\eta_{0} z_{m}}\right)^{2}+\left(\frac{z_{m}^{\prime}}{\eta_{0}}\right)^{2}} .
$$

The longitudinal invariant $I_{L}$ expressed in terms of the total momentum spread is

$$
\frac{1}{2} \eta_{0}^{2} \Delta^{2}+\frac{K_{L}}{z_{m}}=I_{L}
$$

If $z_{m, i}$ is the bunch length at the beginning of drift and $z_{m, f}$ the bunch length at the end of the drift then the conservation of $I_{L}$ results in

$$
\Delta_{i}^{2}+\frac{2 K_{L}}{\eta_{0}^{2} z_{m, i}}=\Delta_{f}^{2}+\frac{2 K_{L}}{\eta_{0}^{2} z_{m, f}}
$$

with $\Delta_{i}$ as the initial total momentum spread at the beginning of the drift and $\Delta_{f}$ as the finally total momentum spread at the end of the drift. For an arbitrary ellipse, we define the modified space charge parameter

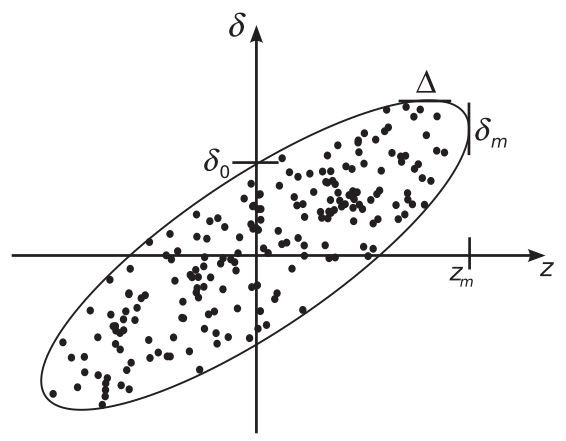

FIG. 3. Rotated ellipse in phase space $\delta, z . \Delta=\delta p / p_{0}$ is the maximum momentum spread, $\delta_{0}=\left(\delta p / p_{0}\right)_{0}$ the maximum momentum deviation in the center, and $\delta_{m}=\left(\delta p / p_{0}\right)_{m}$ the momentum offset at the ends.

$$
\Sigma^{\prime}=\frac{2 K_{L}}{\eta_{0}^{2} z_{m} \Delta^{2}}=\frac{\Sigma}{1+f^{2}}
$$

where $f=\delta_{m} / \delta_{0}=-\hat{\alpha}$ is the ellipse parameter. Equation (13) can be simplified to

$$
1+\Sigma_{i}^{\prime}=\frac{\Delta_{f}^{2}}{\Delta_{i}^{2}}+\frac{\Sigma_{i}^{\prime}}{\chi}
$$

where $\chi=z_{m, f} / z_{m, i}$ is the debunching factor. If we can neglect $\Sigma_{i}^{\prime} / \chi\left(z_{m, f} \gg z_{m, i}\right)$, the finally total momentum spread results as

$$
\Delta_{f}=\Delta_{i} \sqrt{1+\Sigma_{i}^{\prime}}=\sqrt{\Delta_{i}^{2}+\frac{2 K_{L}}{\eta_{0}^{2} z_{m, i}}} .
$$

The minimum momentum spread for the debunched beam is determined through

$$
\Delta_{f} \approx \sqrt{\frac{2 K_{L}}{\eta_{0}^{2} z_{m, i}}}
$$

which depends on the initial bunch length and on the longitudinal perveance only.

In Fig. 4 the two expressions, Eq. (16) ("model") and Eq. (17) ("limit"), are shown together with results from SIS measurements. In our measurement an ${ }^{40} \mathrm{Ar}^{18+}$ beam was injected for one turn with the energy of $11.4 \mathrm{MeV} / \mathrm{u}$ from the UNILAC into the SIS. The injected UNILAC beam consists of microbunches $(36 \mathrm{MHz})$ with initial parameters $\Delta_{i} \approx 0.9 \times 10^{-3}$ and $z_{m, i} \approx 0.2 \mathrm{~m}\left( \pm 55^{\circ}\right)$. The number of ions per microbunch is in the range $N_{b}=$ $1-5 \times 10^{7}$. The momentum spread of the resulting coasting beam was obtained from the longitudinal Schottky

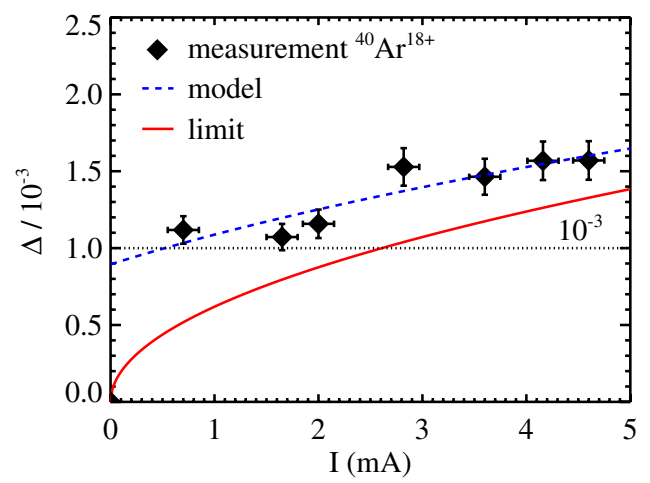

FIG. 4. The final total momentum spread of the debunched, coasting beam in the SIS for ${ }^{40} \mathrm{Ar}^{18+}$ ions as a function of the injected UNILAC current (diamonds). The error bars represent the current fluctuations of the injected beam and the fitting errors to the longitudinal Schottky signal. The measured momentum spread lies above the limit determined by the rf bucket (black dotted line). Equation (16) (blue dashed line) reproduces the measured data very well. For UNILAC currents exceeding $I \approx 2.5 \mathrm{~mA}$, the lower limit of momentum spread given by Eq. (17) (red continuous line) lies above the tolerable limit. 
signal. The error bars in Fig. 4 represent the fitting errors to the longitudinal Schottky bands and the fluctuations of the current injected from the UNILAC. For the SIS intensity upgrade, the resulting total momentum spread of the coasting beam has to remain below $\Delta \approx 2 \sigma_{p}=1 \times 10^{-3}$ because of the available rf bucket area for fast ramping. In Fig. 4 we can see that the measured momentum spread lies above this limit (black dotted line). Equation (16) (blue dashed line) reproduces the measured data very well. The momentum spread increases during the debunching because the space charge energy of the microbunches is transformed into incoherent thermal momentum spread of the beam ions. For UNILAC currents exceeding $I \approx$ $2.5 \mathrm{~mA}$ also the lower limit of momentum spread given by Eq. (17) (red continuous line) lies above the limit. A possible cure would be to further stretch the microbunches before injection.

\section{MICROBUNCH DYNAMICS: LONG TERM}

The envelope equation is valid until the microbunches start to overlap. If the injected microbunches overlap with the adjacent bunches, this leads to the formation of filaments in longitudinal phase space (see Fig. 5). After sufficiently many revolutions, the beam distribution consists of almost parallel filaments [3]. The time $t_{M}$ needed for the formation of $M$ filaments is determined through

$$
M=-2 \eta_{0} \beta_{0} c \frac{\Delta}{l} t_{M},
$$

where $l$ is the distance between the microbunches and $\Delta \approx 2 \sigma_{p}$ the momentum spread. The velocity difference between adjacent filaments is

$$
\Delta v=-2 \eta_{0} \beta_{0} c \frac{\Delta}{M}
$$

The evolution of the filaments can be studies within the longitudinal Vlasov equation

$$
\frac{d f\left(z, v_{z}, t\right)}{d t}=\frac{\partial f}{\partial t}+v_{z} \frac{\partial f}{\partial z}-\frac{q \eta_{0}}{\gamma_{0} m_{0}} E_{z} \frac{\partial f}{\partial v_{z}}=0
$$

for the longitudinal distribution function $f\left(z, v_{z}, t\right) . v_{z}$ is the relative velocity $-\eta_{0} \beta_{0} c \delta$ and $m_{0}$ the ion mass. For $M \gg 1$ the beam distribution can be approximated as

$$
f_{0}\left(v_{z}\right)=\frac{\lambda_{0}}{M B_{f}} \sum_{j=1}^{M} \delta\left(v_{z}-v_{j}\right),
$$

where $v_{j}$ is the velocity of the $j$ th filament, $\lambda_{0}$ the total line density after debunching, and $B_{f}=\lambda_{0} / \lambda_{\max }$ the initial bunching factor of the microbunches. The bunching factor accounts for the fact that the innermost filaments have a larger intensity than the outer filaments due to the initial microbunches distribution.

For a perturbed distribution function $f\left(v_{z}, z, t\right)=$ $f_{0}\left(v_{z}\right)+f_{n}(z, t) e^{i(\omega t-k z)}$, we obtain from the linearized Vlasov equation (20)

$$
f_{n}(z, t)=-i \frac{q \eta_{0}}{\gamma_{0} m_{0}} E_{n} \frac{f_{0}^{\prime}\left(v_{z}\right)}{\omega-k v_{z}} .
$$

The electric field amplitude can be obtained from the line density amplitude and the longitudinal impedance [Eq. (5)] at harmonic $n$ :

$$
E_{n}=-\frac{q \beta_{0} c}{2 \pi R} Z_{\|} \lambda_{n}
$$

The dispersion relation for $M$ filaments is obtained then as

$$
-i \frac{q \eta_{0} I_{0}}{2 \pi \gamma_{0} \beta_{0}^{2} m_{0} c^{2}} \frac{Z_{n}}{n} \frac{n^{2} \omega_{0}^{2}}{M B_{f}} \sum_{j=1}^{M} \frac{1}{\left(\omega-k v_{j}\right)^{2}}=1,
$$

where $I_{0}=q \lambda_{0} \beta_{0} c$ is the current after debunching.

For $M=1, B_{f}=1$, and $n \omega_{0}=k v_{0}=k \beta_{0} c$, one obtains the well-known expression for the coherent frequency shift due to space charge for a beam with no momentum spread (cold beam). For the multistream beam there a $2 M$ space charge waves. With increasing intensity the phase velocities on adjacent filaments overlap and the space charge waves couple with each other. A pair of real solutions for $\omega$ of the dispersion relation Eq. (24) turns into a pair of complex conjugate solutions [3]. The instability threshold can be obtained from the marginal solution $\omega=0$, which corresponds to the coupling of the two innermost filaments at $v= \pm \Delta v / 2$. For all other pairs at $v_{j}= \pm \Delta v(2 j-1) / 2$ with $j>1$, the transition to instability occurs simultaneously. From the dispersion relation Eq. (24) together with the approximation [3]
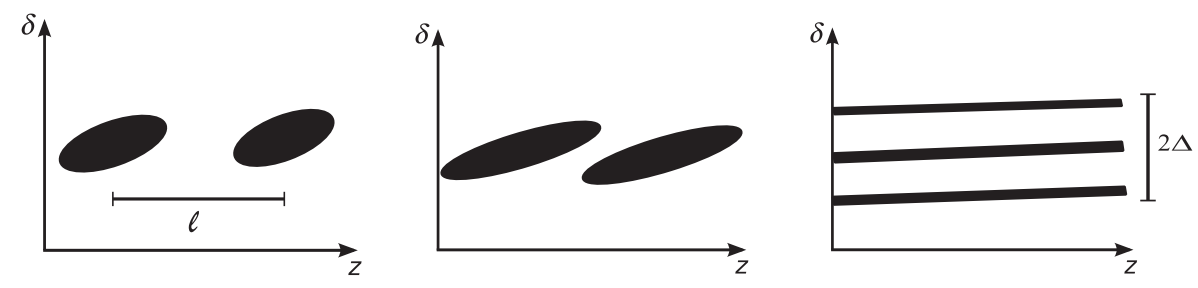

FIG. 5. Debunching of microbunches and subsequent formation of filaments. $l$ is the distance between the microbunches and $\Delta \approx 2 \sigma_{p}$ the momentum spread of the debunched beam. 


$$
\sum_{j=1}^{M}=\frac{1}{(2 j-1)^{2}} \approx \sum_{j=1}^{\infty} \frac{1}{(2 j-1)^{2}}=\frac{\pi^{2}}{8}
$$

one obtains the threshold for the onset of a multistream instability caused by the space charge impedance in terms of the critical number of filaments,

$$
M_{\mathrm{thr}}=\frac{32 B_{f}}{\pi^{2} U_{\mathrm{sc}}}
$$

where

$$
U_{\mathrm{sc}}=\frac{q I_{0} g Z_{0}}{4 \pi \beta_{0}^{3} \gamma_{0}^{3} m_{0} c^{2}\left|\eta_{0}\right| \sigma_{p}^{2}}
$$

is the space charge parameter for a coasting beam in the long wavelength regime $\left(n \ll n_{c}\right)$. For a coasting beam with momentum spread, the space charge parameter represents the ratio of the coherent velocity of the two space charge waves to the incoherent thermal velocity of the beam ions. After debunching the time needed to reach $M_{\text {thr }}$ filaments is

$$
t_{\mathrm{thr}}=\frac{M_{\mathrm{thr}} C}{4 n_{B}\left|\eta_{0}\right| \beta_{0} c \sigma_{p}}
$$

with the ring circumference $C$ and the number of microbunches per turn $n_{B}$.

\section{SIMULATION AND OBSERVATION OF THE MULTISTREAM INSTABILITY}

A longitudinal simulation code has been developed in order to study the evolution of microbunches after injection. The simulation code employs a particle-in-cell (PIC) scheme similarly to the numerical scheme presented in [11]. The code solves the longitudinal equation of motion together with the self-consistent space charge field. The self-consistent space charge field is obtained from Eq. (5) and the Fourier transformed line density. The fast-Fourier transformation is calculated on a one-dimensional grid with the grid spacing $\Delta z$. A large number of macroparticles per cell $\Delta z$ is used in order to reduce the effect of artificial noise (typically more than 1000). The number of cells is chosen as $N_{z}=512$ and the total number of macroparticles is $N_{\mathrm{PIC}}=10^{6}$. In the simulation we only account for a subsection of the SIS ring which includes three microbunches of the total 168 per turn. The time step is kept below $\Delta t<\Delta z / v_{\max }$, where $v_{\max }$ is the maximum relative velocity. The PIC solver together with the space charge routine were validated previously against various analytic examples (see, for example, Refs. [1,11,12]). The present simulation code was validated against Schottky signals and conservation of the total energy (kinetic and space charge).

The evolution of the multistream instability obtained from the simulation is shown in Fig. 6. As an example case we use an ${ }^{40} \mathrm{Ar}^{18+}$ beam with the space charge parameter $U_{\mathrm{sc}}=0.3$ and an initial bunching factor of $B_{f}=$ 0.47 for the microbunches. We only treat the case of a single turn injection. In Fig. 6(b), the formation of $M$ filaments and the onset of the instability between the inner filaments can be seen. Afterwards the transition to a turbulent state with a broadband fluctuation spectrum can be observed [Fig. 6(c)]. Because of the space charge induced multistream instability, the resulting debunched beam exhibits a turbulent, pseudo-Schottky spectrum. In order to verify the instability threshold in the simulations we performed a parameter scan by varying the initial microbunch intensity. All other parameters are kept constant. The waterfall plot in Fig. 7(a) shows the evolution of the total electric field energy $W$ as a function of time and of the initial space charge parameter. The initial energy of the microbunch space charge electric field decreases very fast on a time scale much less than a millisecond. During the subsequent filamentation, the field energy remains very low until the critical number of filaments is reached indicated by the white line [given by Eq. (28)]. Afterwards the electric field energy increases rapidly due to the coherent fluctuations generated by the multistream instability. The instability results in a "turbulent" frequency spectrum with a corresponding persistent electric field energy. For very small $U_{\text {sc }} \ll 1$, we do not observe the onset of the multistream instability in our simulations. In agreement with the analytic results, our simulations show the onset of a fast instability following $t=t_{\mathrm{thr}}$. Figure 7(b) shows the
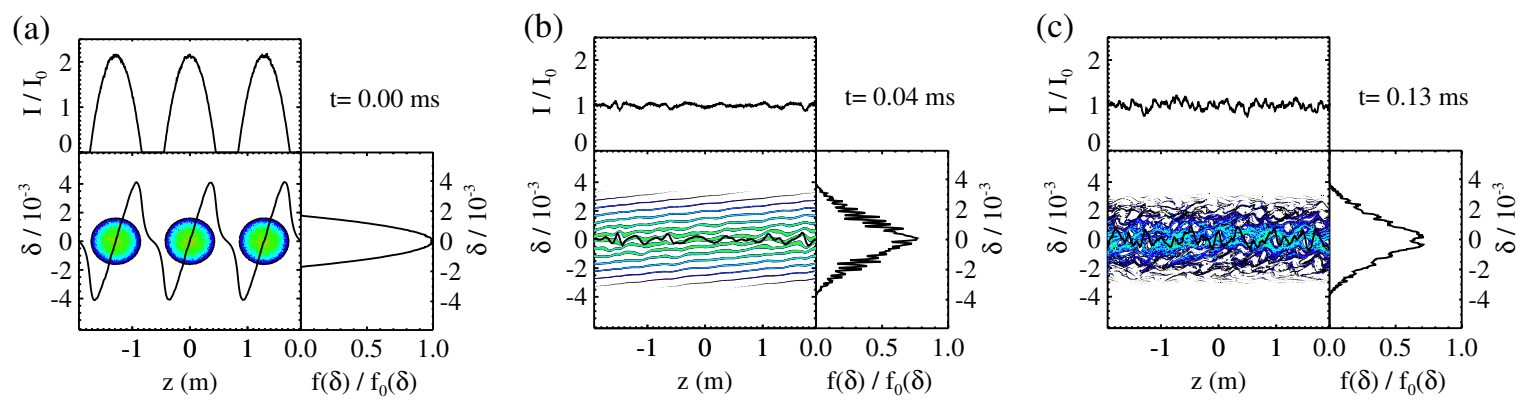

FIG. 6. Snapshots of the simulated time evolution of the multistream instability for an ${ }^{40} \mathrm{Ar}^{18+}$ beam with a space charge parameter of $U_{\mathrm{sc}}=0.3$ for the coasting beam and the bunching factor of $B_{f}=0.47$ for the initial microbunches (a). The onset of the instability between the inner filaments (b) and the fluctuations after saturation (c) is clearly visible. 

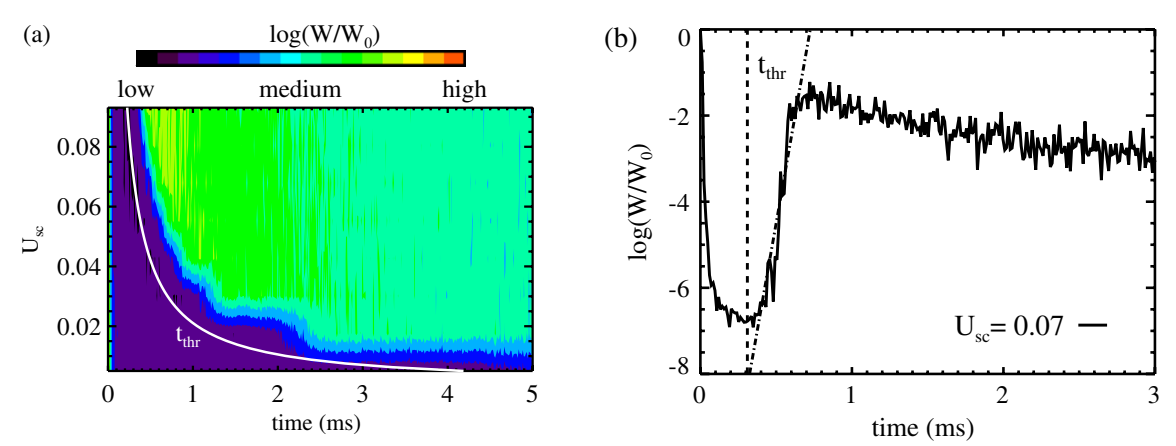

FIG. 7. Simulation scan of the total electric field energy $W$ divided by the initial field energy $W_{0}$ for different microbunch intensities versus time. The white line in (a) indicates the time needed to reach the critical number of filaments given through Eq. (28). The initial bunching factor of the microbunches in the simulation is chosen as $B_{f}=0.42$. Part (b) shows the total electric field energy for $U_{\mathrm{sc}}=0.07$. The time $t_{\mathrm{thr}}=0.31 \mathrm{~ms}$ for this case is indicated by the dashed, vertical line. The dotted dashed curve represents the exponential increase of the multistream instability.
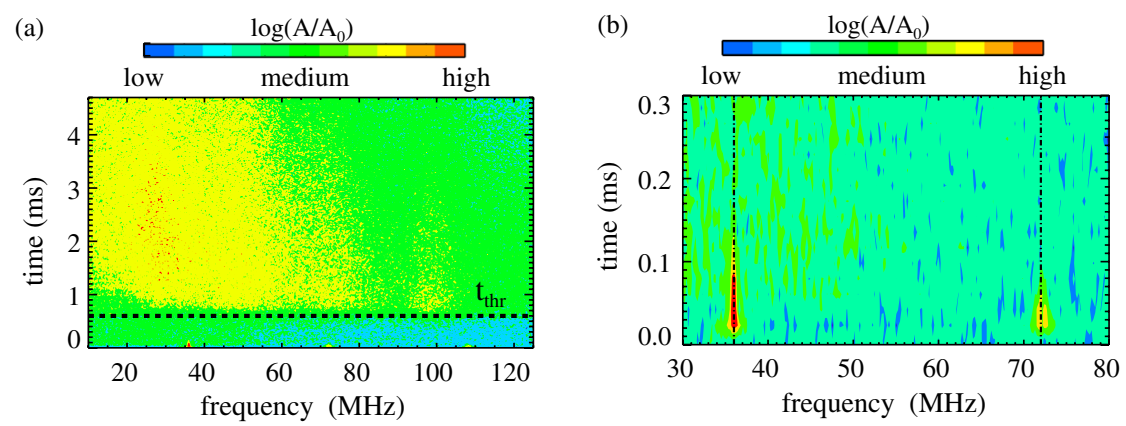

FIG. 8. Measured frequency spectrum after injection into the SIS. The dashed line in (a) indicates the time needed for the formation of the critical number of filaments $t_{\text {thr }}$. In (b), one can observe the $36 \mathrm{MHz}$ structure of the UNILAC microbunches and their higher harmonics.

evolution of the field energy for $U_{\mathrm{sc}}=0.07$. The fast decrease of the initial microbunch space charge energy and the subsequent exponential increase due to the coherent fluctuations generated by the multistream instability are clearly visible.

In our experiments we measure the current profile evolution in the SIS right after injection. Figure 8(a) shows the obtained frequency spectrum during the first $5 \mathrm{~ms}$ for
$N=3 \times 10^{9}{ }^{40} \mathrm{Ar}^{18+}$ ions that were injected within one turn. The momentum spread measured from the Schottky noise after debunching was $\sigma_{p} \approx 10^{-3}$ and the corresponding space charge parameter $U_{\mathrm{sc}}=0.025$. The bunching factor of the injected microbunches is in the range $B_{f} \approx 0.5-0.6$. Figure 8 (b) shows the initial $36 \mathrm{MHz}$ signal of the UNILAC microbunches and their higher harmonics. This signal decays within $0.1 \mathrm{~ms}$ during the debunching.
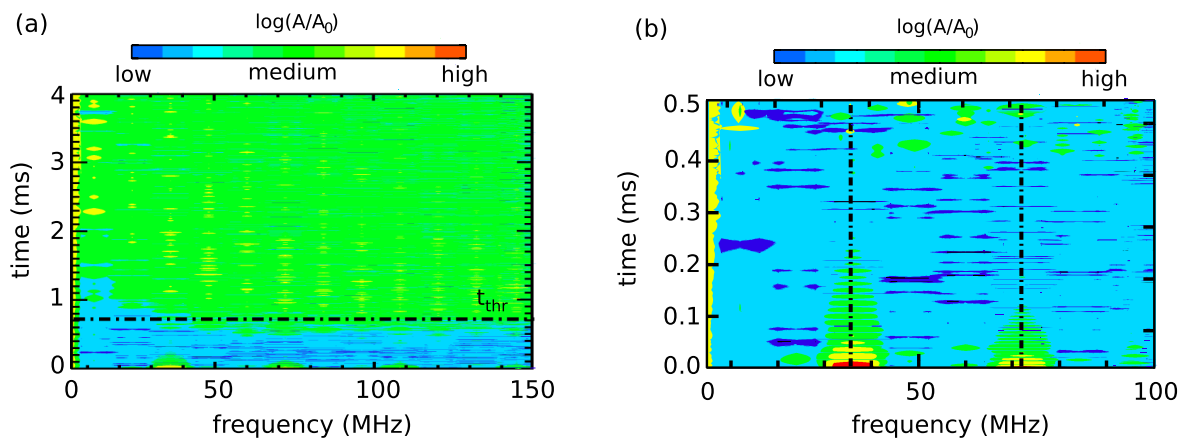

FIG. 9. Simulated frequency spectrum of the ${ }^{40} \mathrm{Ar}^{18+}$ measurement shown in Fig. 8. The dashed line in (a) indicates the time needed for the formation of the critical number of filaments $t_{\mathrm{thr}}$. In (b), the harmonics of the initial microbunches are shown. 
After a time duration $t=t_{\text {thr }}$ which corresponds to the generation of $M_{\text {thr }}$ filaments [dashed line in Fig. 8(a), representing Eq. (28)], one observes a rapid transition to a broad frequency spectrum. In the measured frequency spectrum, the transition to a broad frequency spectrum occurs later for the smallest frequencies $(<25 \mathrm{MHz})$ which is in agreement with the fact that the shortest wavelengths have faster growth rates [3]. The higher fluctuation amplitudes in the frequency range between 10 to $50 \mathrm{MHz}$ are a consequence of the nonlinearity of the longitudinal pickup in this frequency range [13].

The corresponding simulation result is shown in Fig. 9. It shows a similar transition to a broadband fluctuation spectrum after $t=t_{\text {thr }}$ [see Fig. 9(a)]. Also the slower transition at low frequencies is reproduced. The harmonics of the initial microbunches are broader [see Fig. 9(b)] because of the lower frequency resolution in the simulation.

In this study, we present experimental and simulation results for single turn injection. Both the experimental and the simulation studies show that also after injection of up to 5 turns there is a sudden transition to a broad fluctuation spectrum right after the predicted number of filaments has been generated. The detailed analysis of simulation and experimental results for multiturn injection is still ongoing. In the SIS, the frequency ratio between the UNILAC injection frequency $(36 \mathrm{MHz}$ ) and the revolution frequency $(\approx 215 \mathrm{kHz})$ is not an integer. Therefore the freshly injected microbunches do not exactly overlap with the previously injected ones, like in the PSR studies [2]. In our simulations, after injection of many turns $(>10)$ and if we exactly match the frequency ratio between the UNILAC and the SIS, we do observe the formation of large, persistent phase space holes similar to Ref. [2] and not the multistream instability.

\section{PARASITIC COHERENT ENERGY LOSS}

In addition to the space charge impedance also resistive impedance components are present. In this section we will briefly analyze the possible interplay of the space charge driven multistream instability and the microwave instability, driven by the broadband impedance of the machine. The broadband impedance summarizes the individual impedance contribution from cavitylike structures and other geometry variations of the beam pipe. The broadband impedance can be represented as

$$
Z_{n}^{b b}=\frac{R_{s}}{1+i Q\left(\frac{\omega}{\omega_{r}}-\frac{\omega_{r}}{\omega}\right)}
$$

with the shunt impedance $R_{s}$, the quality factor $Q \approx 1$, the eigenfrequency $\omega_{r}$, and $\omega_{n}=n \omega_{0}$. The eigenfrequency is chosen as the cutoff frequency of the beam pipe $\omega_{r} \approx$ $c /(2 \pi b)$. At resonance, assuming $Z_{s} \gg R_{s}$ and a coasting cold beam, one can easily obtain the instability growth rate $[7,14]$

$$
\tau_{I}^{-1} \approx R_{s} \sqrt{\frac{q I_{0}\left|\eta_{0}\right|}{8 \pi R^{2} \gamma_{0} m_{0}}\left|\frac{n}{Z_{n}^{\mathrm{sc}}}\right|}
$$

This expression applies for the microwave instability in a single filament. For a multistream beam, the above expression can serve as an upper limit for the instability growth rate. For $\tau_{I}<t_{\text {thr }}$ the microwave instability can develop before the multistream instability. In our simulation we used an example case with a shunt impedance of $R_{s} / n \approx$ $25 \Omega$ and a corresponding microwave instability growth rate $\tau_{I}^{-1}=0.06 \mathrm{~ms}$, which is smaller than the time that is needed to reach the critical number of filaments $t_{\mathrm{thr}} \approx$ $0.1 \mathrm{~ms}$ for the multistream instability. Figure 10 shows the evolution of the distribution function. The deformation of the initial electric field of the microbunch caused by the broadband impedance is clearly visible [Fig. 10(a)]. Still one can observe the onset of the multistream instability between the inner filaments at $t=0.12 \mathrm{~ms}$ [Fig. 10(b)] and a subsequent broad fluctuation spectrum after $1 \mathrm{~ms}$ [Fig. 10(c)]. The evolution of the relative momentum shift $\Delta_{p_{0}}$ and of the momentum spread $\Delta$ are shown in Fig. 11. The relationship between the relative momentum shift and the energy loss is

$$
\Delta_{p_{0}}=\frac{\Delta p_{0}}{p_{0}}=\frac{1}{\beta_{0}^{2}} \frac{\Delta E_{0}}{E_{0}},
$$
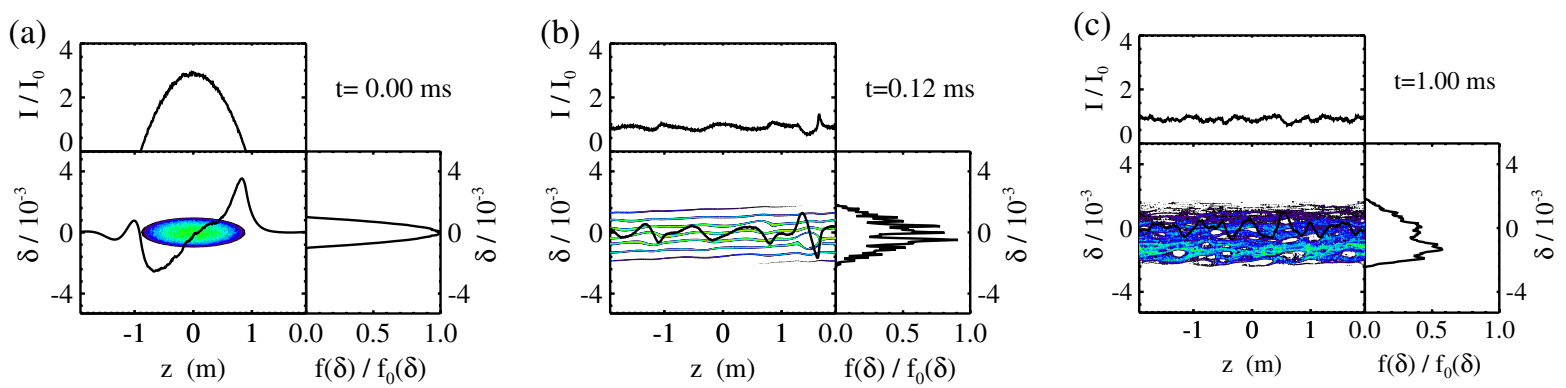

FIG. 10. Snapshots of the simulated time evolution of multistream instability and the effect of a broadband resonator impedance. In (a), the deformation of the initial electric field caused by the impedance is clearly visible. Still one can observe the onset of the multistream instability between the inner filaments (b) and a subsequent broad fluctuation spectrum after $1 \mathrm{~ms}$ (c). 

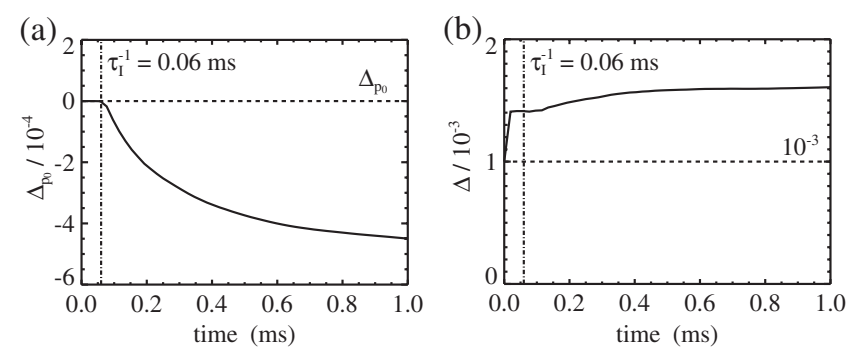

FIG. 11. Multistream instability and the effect of a broadband resonator impedance. Part (a) shows as a solid curve the simulated energy loss and (b) the momentum spread evolution. In both figures the dotted dashed, vertical line presents the microwave instability growth rate.

where $p_{0}$ is the design momentum and $\Delta p_{0}$ is the shift in the average momentum of the beam. One observes that the final momentum spread is still dominated by the transformation of the initial microbunch space charge into kinetic energy. However, in the presence of a broadband impedance we observe a slowing down of the beam. The interaction of the persistent fluctuation spectrum with the broadband impedance causes a momentum loss, which is of the same order as the final momentum spread. The result can be understood in terms of the parasitic energy loss of the beam per turn [15]:

$$
\overline{\Delta E}=-2 \pi q^{2} N_{b} \omega_{0} \sum_{n=-\infty}^{\infty}\left|\tilde{\lambda}_{n}\right|^{2} \operatorname{Re} Z_{\|}\left(n \omega_{0}\right)
$$

For a broadband impedance the momentum loss per turn can be obtained as

$$
\bar{\Delta}_{p_{0}}=-\frac{2 \pi q^{2} N_{b} \omega_{0} R_{s}}{\beta_{0}^{2} \gamma_{0} m_{0} c^{2}} \sum_{n=-\infty}^{\infty}\left|\tilde{\lambda}_{n}\right|^{2}
$$

We confirmed the validity of Eq. (33) within our simulations for the chosen parameters of the broadband impedance. In the SIS experiments we observed an energy loss after the injection. The energy loss relative to the injection energy is obtained from the averaged Schottky spectrum $t \approx 200-500 \mathrm{~ms}$ after injection. The results of

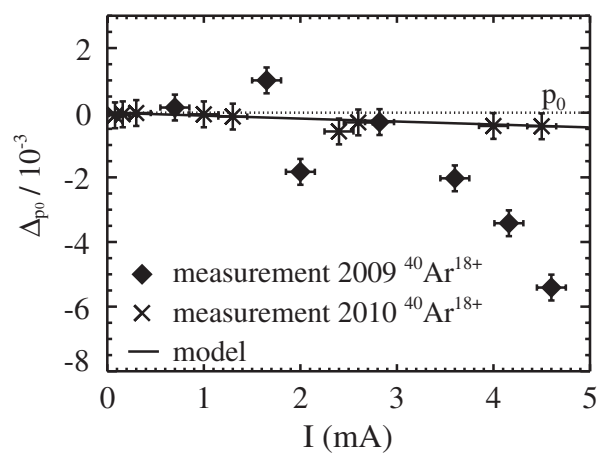

FIG. 12. Comparison of measured energy loss in the SIS with the model of parasitic coherent energy loss as a function of intensity. Shown are the 2009 measurement results (diamonds) and the 2010 results (crosses) together with an analytic estimate. two measurements performed in 2009 and in 2010 are shown in Fig. 12. The later measurement was preformed as part of the work presented in Ref. [16]. The solid line corresponds to the momentum loss obtained from Eq. (33) using $R_{s} / n=50 \Omega$, an assumed fluctuation amplitude of $\sum_{n=-\infty}^{\infty}\left|\tilde{\lambda}_{n}\right|=0.1$, and a lifetime of the fluctuations of $T=500 \mathrm{~ms}$. The 2010 measurement results agree very well with this simple model. For the 2009 measurements we observe a strong deviation from the model. A possible explanation for the disagreement could be a systematic misadjustment of the current-dependent settings of the buncher cavities in the transfer line to the SIS. Although the assumptions made are rather crude, the multistream instability right after injection followed by a broad fluctuation spectrum, which interacts with the broadband impedance of the machine is a possible explanation of the observed intensity dependent energy loss observed in the SIS.

\section{CONCLUSIONS}

The space charge induced multistream instability during the debunching of intense linac microbunches is studied using analytical and numerical models as well as experimental observations in the SIS synchrotron at GSI. The initial condition for the multistream instability is determined by the injected microbunches and their space charge field. The initial space charge field determines the momentum spread of the debunched beam. An analytic model for the multistream instability threshold for the debunched beam is presented. The predicted threshold can be reproduced very well with a longitudinal simulation code. The application of the purely longitudinal space charge model is justified even for microbunches with a transverse offset. In the SIS, the multistream instability can be identified in the measured fluctuation spectrum right after the debunching of the injected microbunches. There are also indications that the turbulent broadband fluctuation spectrum causes a slowing down of the debunched beam, which affects the efficiency of the subsequent adiabatic rf capture. The present study focuses on the multistream instability after injection one or a few turns. Future work will also address the two limiting cases (large persistent structures 
versus multistream instability) during multiturn injection in the SIS.

\section{ACKNOWLEDGMENTS}

The authors acknowledge Thomas Weiland for his support, Lars Groening and Stefan Paret for their help during the experiments, and Giuliano Franchetti and Ingo Hofmann for fruitful discussions.

\section{APPENDIX: LONGITUDINAL SPACE CHARGE FIELD FOR A BUNCH WITH A TRANSVERSE OFFSET}

In this section we will calculate the longitudinal electric field for a homogeneous, round beam with a transverse offset $d$ and radius $a$ in a circular vacuum chamber with radius $b$. Similar to the centered beam $[7,17,18]$, the longitudinal electric field for a beam with an offset can be evaluated by applying Faraday's law

$$
\oint \vec{E} \cdot d \vec{l}=-\frac{\partial}{\partial t} \int \vec{B} \cdot d \vec{A}
$$

along the rectangular path in Fig. 13. The transverse electric field for a displaced beam in a circular vacuum chamber was obtained in $[19,20]$ using the method of images charges,

$$
\vec{E}_{r}(\vec{r})=\vec{E}_{\mathrm{sf}}(\vec{r})+\vec{E}_{\mathrm{im}}(\vec{r})
$$

where the transverse self-fields (sf) and image fields (im) outside the beam are given by

$$
\vec{E}_{\mathrm{sf}}(\vec{r})=\frac{q \lambda}{2 \pi \epsilon_{0}} \frac{\vec{r}-\vec{r}_{0}}{\left|\vec{r}-\vec{r}_{0}\right|^{2}} ; \quad \vec{E}_{\mathrm{im}}(\vec{r})=\frac{-q \lambda}{2 \pi \epsilon_{0}} \frac{\vec{r}-\vec{r}_{0}^{\prime}}{\left|\vec{r}-\vec{r}_{0}^{\prime}\right|^{2}}
$$

and inside the beam by

$$
\vec{E}_{\mathrm{sf}}(\vec{r})=\frac{q \lambda}{2 \pi \epsilon_{0}} \frac{\vec{r}-\vec{r}_{0}}{a^{2}} ; \quad \vec{E}_{\mathrm{im}}(\vec{r})=\frac{-q \lambda}{2 \pi \epsilon_{0}} \frac{\vec{r}-\vec{r}_{0}^{\prime}}{\left|\vec{r}-\vec{r}_{0}^{\prime}\right|^{2}} .
$$

Here $\vec{r}_{0}^{\prime}=\left(b^{2} /\left|\vec{r}_{0}\right|^{2}\right) \vec{r}_{0}$ is the distance between the axis and the image beam, where $\vec{r}_{0}$ is the distance between the axis and the displaced beam. According to Fig. 13

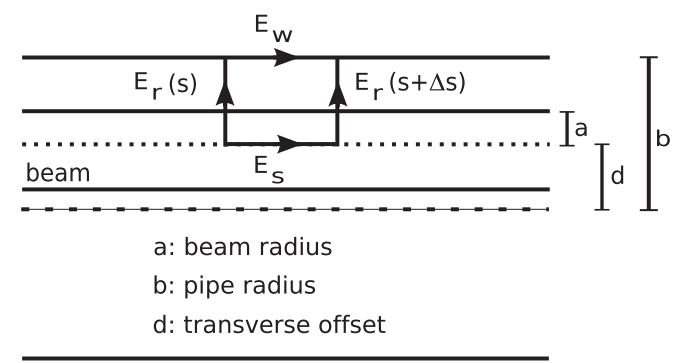

FIG. 13. Field configuration and integration path for the calculation of the longitudinal electric field for a uniform, round beam with a transverse offset $d$ and a radius $a$ in a circular vacuum chamber with radius $b$. we have $\vec{r}_{0}=d \vec{e}_{r}$ and $\vec{r}_{0}^{\prime}=\frac{b^{2}}{d} \vec{e}_{r}$. For the line integral in Eq. (A1) follows

$$
\begin{aligned}
\oint \vec{E} \cdot d \vec{l}= & E_{s} \Delta s+\frac{\Delta \lambda}{2 \pi \epsilon_{0}}\left[\int_{d}^{a+d} \frac{r-d}{a^{2}} d r-\int_{d}^{a+d} \frac{d r}{r-b^{2} / d}\right. \\
& \left.+\int_{a+d}^{b} \frac{d r}{r-d}-\int_{a+d}^{b} \frac{d r}{r-b^{2} / d}\right] \\
= & E_{s} \Delta s+\frac{q g_{\text {off }}}{4 \pi \epsilon_{0}} \Delta \lambda
\end{aligned}
$$

where $\Delta \lambda=\lambda(s+\Delta s)-\lambda(s)$ is the line density difference. The geometry factor for a displaced beam is obtained as

$$
\begin{aligned}
g_{\text {off }}= & 2\left(\frac{(a+d)^{2}}{2 a^{2}}-\frac{d}{a}-\frac{d^{2}}{2 a^{2}}+\ln \left[\frac{d-b^{2} / d}{a+d-b^{2} / d}\right]\right. \\
& \left.+\ln \left[\frac{b-d}{a}\right]+\ln \left[\frac{a+d-b^{2} / d}{b-b^{2} / d}\right]\right) \\
= & 1+2 \ln \left[\frac{b+d x_{d}}{a}\right],
\end{aligned}
$$

where the scale factor is

$$
x_{d}=-\frac{d}{b}
$$

The offset $d$ enters only through the $g$ factor's logarithm and causes a smaller effective beam pipe radius. In the limit value $d=0$, the geometry factor for a centered beam is obtained:

$$
g_{\text {off }}(d=0)=1+2 \ln \frac{b}{a} .
$$

For the magnetic field the corresponding image current is used to evaluate the magnetic flux on the rhs of Faraday's law $[7,17]$ and we obtain the longitudinal electric field for a displaced beam as

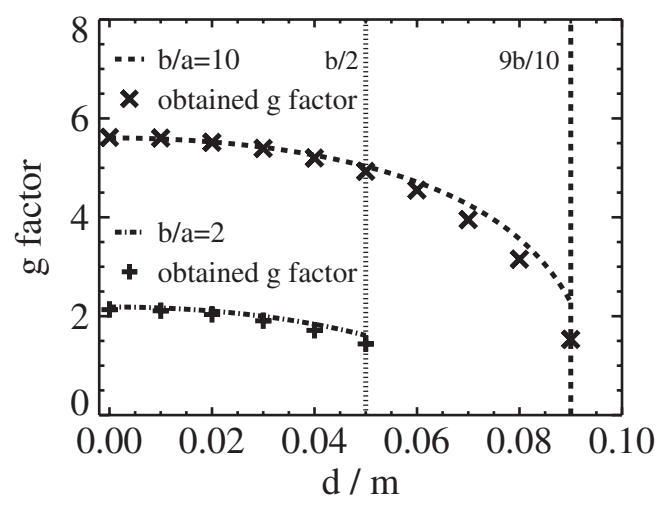

FIG. 14. The $g$ factor (symbols) obtained from the numerical electric field as a function of the transverse offset $d$ for two different ratios of pipe radius to beam radius. The dashed curves show Eq. (A6). 


$$
E_{z}=-\frac{q g_{\text {off }}}{4 \pi \epsilon_{0} \gamma_{0}^{2}} \frac{\partial \lambda}{\partial z}
$$

with $z=s-s_{0}$. For a parabolic microbunch with the bunch length $z_{m}=0.15 \mathrm{~m}$ the analytic solution for the electric field Eq. (A9) is compared to the numerically obtained longitudinal field from the 3D Poisson solver in CST EM STUDIO ${ }^{\circledR}$. For different transverse offsets $d$ we obtain the $g$ factor from the numerically obtained longitudinal electric field along the bunch axis. The results for two $b / a$ ratios are shown in Fig. 14. The analytical as well as the numerical study confirms that for the bunch parameters of interest the longitudinal electric field depends only logarithmically on the transverse bunch offset $d$. Therefore a longitudinal model is sufficient in order to describe the longitudinal dynamics of the microbunches.

[1] O. Boine-Frankenheim and I. Hofmann, Phys. Rev. ST Accel. Beams 3, 104202 (2000).

[2] S. Cousineau, V. Danilov, J. Holmes, and R. Macek, Phys. Rev. ST Accel. Beams 7, 094201 (2004).

[3] I. Hofmann, Part. Accel. 34, 211 (1990).

[4] S. Paret and O. Boine-Frankenheim, in Proceedings of the HB2010 Conference, Morschach, Schwitzerland, 2010, p. 72.

[5] O. Boine-Frankenheim, in Proceedings of the IPAC'10 Conference, Kyoto, Japan (ICR, Kyoto, 2010), Vol. 10, p. 2430.
[6] M. G. Minty and F. Zimmermann, Measurement and Control of Charged Particle Beams (Springer-Verlag, Berlin, 2003).

[7] M. Reiser, Theory and Design of Charged Particle Beams, Wiley Series in Beam Physics and Accelerator Technology (Wiley-VCH, Berlin, 2008).

[8] A. M. Al-Khateeb, O. Boine-Frankenheim, I. Hofmann, and G. Rumolo, Phys. Rev. E 63, 026503 (2001).

[9] D. Neuffer, IEEE Trans. Nucl. Sci. 26, 3031 (1979).

[10] G. Franchetti, I. Hofmann, and G. Rumolo, Phys. Rev. ST Accel. Beams 3, 084201 (2000).

[11] O. Boine-Frankenheim and T. Shukla, Phys. Rev. ST Accel. Beams 8, 034201 (2005).

[12] A. Al-Khateeb, O. Boine-Frankenheim, R. W. Hasse, and I. Hofmann, Phys. Rev. ST Accel. Beams 6, 014205 (2003).

[13] U. Schaaf, Ph.D. dissertation, Universität Frankfurt, 1991.

[14] F. J. Sacherer, IEEE Trans. Nucl. Sci. 20, 825 (1973).

[15] K. Y. Ng, Physics of Intensity Dependent Beam Instabilities (World Scientific, Singapore, 2006).

[16] S. Paret, V. Kornilov, O. Boine-Frankenheim, and T. Weiland, Phys. Rev. ST Accel. Beams 13, 022802 (2010).

[17] V. K. Neil and A. M. Sessler, Rev. Sci. Instrum. 36, 429 (1965).

[18] J. G. Wang, H. Suk, D. X. Wang, and M. Reiser, Phys. Rev. Lett. 72, 2029 (1994).

[19] E. Regenstreif, CERN Report No. CERN/PS/DL 76-7, 1976.

[20] M. A. Furman, Phys. Rev. ST Accel. Beams 10, 081001 (2007). 